\title{
Biomarkers of neoadjuvant/adjuvant endocrine therapy for ER- positive/HER2-negative breast cancer
}

\section{Takayuki Ueno}

Breast Surgical Oncology, Breast Oncology Center, The Cancer Institute Hospital of JFCR, Tokyo, Japan

Correspondence to: Takayuki Ueno. Breast Surgical Oncology, Breast Oncology Center, The Cancer Institute Hospital of JFCR, 3-8-31 Ariake Kotoku, Tokyo 135-8550, Japan. Email: takayuki.ueno@jfcr.or.jp.

\begin{abstract}
Endocrine therapy is one of the key therapeutic components for patients with hormone receptorpositive early stage breast cancer. A lot of efforts have been made in order to explore biomarkers to select optimal endocrine treatment and optimal duration of the treatment. Estrogen receptor (ER) is the most intensively-studied and well-established biomarker for selection of endocrine treatment. Currently, a number of other markers including conventional immunohistochemical markers and molecular markers such as genetic markers and multigene assays have been investigated. Although the clinical utility of PgR expression has been tested in a number of clinical trials of neoadjuvant/adjuvant endocrine therapy, no validated results have been obtained. Oncotype DX Recurrence Score has been reported to be associated with benefit of adjuvant tamoxifen use and the clinical response to neoadjuvant endocrine therapy but more powerful tool is desired for clinical use to optimize endocrine therapy. Neoadjuvant endocrine therapy is considered as a promising strategy to explore biomarkers for endocrine responsiveness as well as to develop a new treatment option in combination with molecular target agents and to study mechanisms underlying endocrine response and resistance. In this manuscript, current understanding on biomarkers of neoadjuvant/adjuvant endocrine therapy for both predictive and prognostic utilities is discussed.
\end{abstract}

Keywords: Hormone receptor; endocrine therapy; neoadjuvant therapy; preoperative endocrine prognostic index (PEPI)

Submitted Jun 03, 2020. Accepted for publication Jun 19, 2020.

doi: $10.21037 / \mathrm{cco}-20-165$

View this article at: http://dx.doi.org/10.21037/cco-20-165

\section{Introduction}

Endocrine therapy is an essential component of the neoadjuvant/adjuvant treatment for hormone receptor -positive early stage breast cancer regardless of menopausal status. Tamoxifen is a major endocrine treatment option, particularly for premenopausal women, and aromatase inhibitors including anastrozole, letrozole and exemestane are another major option for postmenopausal women. The differential use of different types of endocrine therapies is discussed by other authors. Here in this paper, biomarkers for neoadjuvant or adjuvant endocrine therapy will be discussed including both conventional and molecular markers.

\section{Conventional marker}

Estrogen receptor (ER)

ER is the main determinant for clinical use of adjuvant endocrine therapy. The role of ER content as a predictor of response to endocrine therapy has been examined and validated by a number of studies (1). The meta-analysis of the trials of 5 years of adjuvant tamoxifen by Early Breast Cancer Trialists' Collaborative Group (EBCTCG) was reported (2). When the quantitative ER measurement was 
poor (less than $10 \mathrm{fmol} / \mathrm{mg}$ cytosol protein), there was no apparent benefit from adjuvant tamoxifen [relative risk (RR) $0.97 ; 2 \mathrm{P}=0.6]$. However, if $\mathrm{ER}$ was positive (equal to or more than $10 \mathrm{fmol} / \mathrm{mg}$ cytosol protein), the addition of tamoxifen provided substantial benefit $(2 \mathrm{P}<0.00001)$ and the proportional effect depended slightly on quantitative ER measurement: RR 0.67 for ER $10-19 \mathrm{fmol} / \mathrm{mg}$; RR 0.52 for $\mathrm{ER} \geq 200 \mathrm{fmol} / \mathrm{mg}$ (2).

In NSABP B-14 trial, where adjuvant tamoxifen was tested in patients with node-negative hormone receptorpositive breast cancer, quantitative ER mRNA expression was predictive of tamoxifen benefit with a significant interaction in terms of distant recurrence-free survival (DRFS) $(\mathrm{P}<0.001)(3)$. Increased benefit of tamoxifen treatment was observed with increasing levels of ESR1 expression: hazard ratio $(\mathrm{HR})=1.2(95 \%$ CI: $0.72-2.02)$ for lower tertile; HR $=0.59$ (95\% CI: 0.32-1.09) for intermediate; HR $=0.39$ (95\% CI: $0.2-0.77$ ) for higher tertile (3).

Several studies have shown the superiority of aromatase inhibitors over tamoxifen in postmenopausal women with hormone receptor-positive breast cancer. In a substudy from ATAC trial (transATAC) where anastrozole was compared with tamoxifen, ER level did not have any interaction with treatment for time to recurrence (4). In BIG 1-98 trial in which letrozole was compared with tamoxifen, no clear differential effect between treatments was observed according to centrally assessed ER expression level with $\mathrm{P}=0.12$ for interaction although patients with ER-negative disease had HR $=1.32$ (95\% CI: 0.63-2.78) compared with HR $=0.72$ (95\% CI: 0.60-0.86) for those with ER-positive disease (5). Similarly, in TEAM trial in which exemestane was compared with tamoxifen, no statistically significant treatment-by-marker effect of ER expression was observed after adjustment for relevant markers $(\mathrm{P}=0.2)(6)$. Thus, as long as ER is expressed, ER expression level is not a predictor for benefit of aromatase inhibitors over tamoxifen.

In agreement with adjuvant settings, ER Allred expression scores had a linear relationship with response rates of both letrozole and tamoxifen in P024 neoadjuvant study ( $\mathrm{P}=0.0013$ and 0.0061 , respectively) (7) and clinical response to neoadjuvant endocrine therapy is reported to be associated with prognosis (8).

\section{Progesterone receptor (PgR)}

The meta-analysis of 5 years of adjuvant tamoxifen by
EBCTCG showed that the PgR measurement was not predictive of who would respond to tamoxifen in ERpositive disease (2). In ER-negative disease, PgR-positivity gave $\mathrm{RR}$ of 0.90 with no significant benefit from tamoxifen $(2 \mathrm{P}=0.35)(2)$.

Consistently, in NSABP B-14, PgR mRNA expression was not predictive of tamoxifen benefit (3).

In the transATAC study, there was no significant interaction between $\mathrm{PgR}$ and treatment (anastrozole $v s$. tamoxifen) for time to recurrence (4). In addition, anastrozole gave similar benefit over tamoxifen regardless of centrally assessed PgR status in the study: $\mathrm{HR}=0.72$ for PgR-positive and HR $=0.68$ for PgR-negative (4). In BIG 1-98 trial, centrally assessed $\mathrm{PgR}$ expression did not affect the relative efficacy of letrozole over tamoxifen among patients with centrally assessed ER-positive breast cancer (5). Patient with ER-positive breast cancer had better diseasefree survival with letrozole than with tamoxifen regardless of PgR-positivity: $\mathrm{HR}=0.70$ for PgR-positive disease and HR $=0.84$ for PgR-negative disease (5). In TEAM trial where exemestane was compared with tamoxifen, no treatmentby-marker effect for $\mathrm{PgR}$ was observed for exemestane $v s$. tamoxifen $[\mathrm{HR}=0.83 ; 95 \% \mathrm{CI}: 0.65-1.05$ for PgR-rich (Allred score $\geq 5$ ); HR $=0.85$; 95\% CI, 0.61-1.19 for PgRpoor (Allred score $\leq 4$ ); interaction, $\mathrm{P}=0.88$ ] (6). In the metaanalysis conducted by EBCTCG, superiority of aromatase inhibitors to tamoxifen was shown regardless of $\mathrm{PgR}$ expression: RR for ER-positive and PgR-positive disease was 0.74 (95\% CI: 0.64-0.84) and that for ER-positive and PgR-negative disease was 0.57 (95\% CI: 0.45-0.73) (9).

In TEXT and SOFT trials, which are randomized phase III trials investigating adjuvant endocrine therapy for pre-menopausal patients with hormone receptorpositive early breast cancer, lower $\mathrm{PgR}$ expression by immunohistochemistry (IHC) was associated with reduced breast cancer-free interval and seemed to show a greater 5 -year absolute benefit of exemestane + ovarian function suppression (OFS) versus tamoxifen with or without OFS by the non-parametric sliding-window subpopulation treatment effect pattern plot (STEPP) analysis (10). However, there was no interaction between $\mathrm{PgR}$ and any combination of treatment $(\mathrm{P}>0.4)$ and, thus, the clinical significance of $\mathrm{PgR}$ expression for treatment selection has not been established even in pre-menopausal settings.

\section{PEPI}

PEPI stands for preoperative endocrine prognostic index, 
which has been generated for prognostic prediction using samples from clinical trials of neoadjuvant endocrine therapy (11). By using Cox proportional hazards, four factors were selected that were associated with relapsefree survival (RFS) and breast cancer-specific survival (BCSS) in 158 women enrolled in the P024 neoadjuvant endocrine therapy trial, which compared letrozole and tamoxifen before surgery. Four factors were determined using post-endocrine surgical specimen, which included pathological tumor size, nodal status, Ki67 level, and ER status (11). Patients with PEPI score 0 showed a low risk of recurrence and were considered to get less benefit from adjuvant chemotherapy. The index was, then, validated by an independent study of 203 women in the IMPACT trial where anastrozole, tamoxifen, or the combination was compared (11). The prognostic utility of PEPI score was, then, confirmed by ACOSOG Z1031B study, in which neoadjuvant endocrine therapy with anastrozole, exemestane, or letrozole was given to postmenopausal patients (12). After median follow-up of 5.5 years, 3.7\% of the patients with PEPI 0 experienced relapse while $14.4 \%$ of the patients with PEPI $>0$ had recurrence $(\mathrm{HR}=0.27$; 95\% CI: 0.092-0.764; $\mathrm{P}=0.014$ ).

Because selective estrogen receptor degrader (SERD) down-regulates ER expression, modified PEPI (mPEPI) was generated for SERD by excluding post-treatment ER status and showed a similar prognostic power to the original PEPI: after median follow-up of 62.5 months, no patients with mPEPI 0 experienced recurrence in the combined P024 and POL (neoadjuvant letrizole) trials (13).

Currently, a number of clinical trials utilize PEPI or mPEPI as surrogate endpoint for neoadjuvant endocrine therapy with or without molecular target agents (14).

\section{Genetic markers}

\section{ESR1 mutation}

ESR1 is the gene that encodes ER $\alpha$. ESR1 mutation was reported to confer endocrine resistance in breast cancer (15). Some studies have shown that ESR1 mutation, especially mutations in the ligand-binding domain, creates constitutive active state, leading to poor sensitivity of endocrine therapies (15-17). However, it is not clear whether ESR1 mutation in primary breast cancer is associated with endocrine resistance in neoadjuvant/adjuvant settings. In CARMINA 02 trial where neoadjuvant anastrozole and fulvestrant were compared, the frequency of baseline ESR1 mutation was too low (3.4\%) to draw any conclusion regarding endocrine responsiveness (18). In BIG1-98 trial where adjuvant endocrine therapy with letrozole, tamoxifen and a sequential strategy was compared in postmenopausal women, the frequency of ESR1 mutation was reported to be $0 \%$ (19). Similarly, only $3 \%$ $(6 / 183)$ of the primary breast tumor had ESR1 mutation in patients enrolled in BOLERO2 trial (15). Consistently, $3.5 \%(11 / 313)$ and $2.5 \%(7 / 270)$ of the primary breast tumors treated at Memorial Sloan Kettering Cancer Center and Kumamoto University Hospital, respectively, have been reported to have ESR1 mutation $(20,21)$. Indeed, ESR1 mutations were not identified in any of the patients with early-stage ER-positive breast cancer who received neoadjuvant endocrine therapy and showed poor response (PEPI score of $\geq 4)(22)$. In addition, ESR1 mutation was reported to be associated with better recurrencefree survival with no difference in overall survival in patients treated with tamoxifen monotherapy (23). Thus, it remains to be elucidated whether ESR1 mutation is associated with resistance or responsiveness to neoadjuvant/ adjuvant endocrine therapy.

\section{PIK3CA mutation}

PIK3CA encodes the p110- $\alpha$ subunit of the phosphatidylinositol 3-kinase enzyme complex. PIK3CA mutation is the most frequently detected mutation in hormone receptor-positive breast cancers. There have been some controversies on the role of PIK3CA mutation in responsiveness to endocrine therapy.

In the adjuvant setting, no interaction was reported between PIK3CA mutation status and tamoxifen benefit (24). In BIG198 study, PIK3CA mutations were the most common (49\%) among 287 cancer genes of Foundation Medicine's T5targeted panel using next-generation sequencing (19). In the study, PIK3CA mutations were significantly associated with reduced risk of distant recurrence ( $\mathrm{HR}=0.57 ; 95 \%$ CI: 0.38-0.85; $\mathrm{P}=0.006$ ) (19). In addition, patients with PIK3CA mutation (kinase or helical domains) showed a greater magnitude of benefit with adjuvant letrozole over tamoxifen $(\mathrm{HR}=0.18 ; 95 \% \mathrm{CI}: 0.06-0.50)$ than those without mutation ( $\mathrm{HR}=1.26$; 95\% CI: $0.65-2.45)$ with significant interaction $(\mathrm{P}=0.002)(19)$. In TEXT trial where adjuvant exemestane was compared with adjuvant tamoxifen in premenopausal women with OFS, PIK3CA mutation was found in $39.8 \%$ of the examined tumors and associated with improved distant relapse-free survival although it was not an independent marker for prognosis (25). 
Table 1 Neoadjuvant endocrine therapy and Oncotype DX Recurrence Score

\begin{tabular}{|c|c|c|c|c|c|c|c|c|c|}
\hline \multirow{2}{*}{ Agent } & \multirow{2}{*}{ Therapy } & \multirow{2}{*}{$\begin{array}{l}\text { Patient } \\
\text { number }\end{array}$} & \multicolumn{6}{|c|}{ RS } & \multirow{2}{*}{ Reference } \\
\hline & & & Low & Intermediate & High & Low & Intermediate & High & \\
\hline Akashi-Tanaka & Tamoxifen or anastrozole & 43 & 63.6 & 31.3 & 31.3 & N.R. & N.R. & N.R. & (34) \\
\hline JFMC34 & Exemestane & 64 & 59.4 & 58.8 & 20.0 & 90.6 & 76.5 & 46.7 & (35) \\
\hline
\end{tabular}

N.R., not reported.

In addition, there was no significant differences in the effect of PIK3CA mutations between patients treated with exemestane and those with tamoxifen (25). Thus, although it is intriguing to consider PIK3CA mutation as selection marker for aromatase inhibitor over tamoxifen, further studies are necessary to make any conclusion on the clinical use of PIK3CA mutation for treatment selection.

Using samples from two neoadjuvant aromatase inhibitor trials, POL and ACOSOG Z1031, PIK3CA mutation was shown not to associate with clinical response (26). In addition, PIK3CA mutation status did not predict change in Ki67 after 2 weeks of aromatase inhibitor treatment (27). Contradictorily, in phase II CARMINA 02 trial of neoadjuvant comparison between anastrozole and fulvestrant, PIK3CA was significantly more frequently mutated in radiological non-responders than in responders (60.8 vs. $31.6 \%$ ) (18). Because studies on PIK3CA mutation yielded conflicting results in terms of endocrine responsiveness, further clinical research is required.

\section{Multigene assays}

\section{Oncotype DX}

Oncotype DX Recurrence Score (RS) has been developed and shown to be able to assess recurrence risk in patients with hormone receptor-positive early breast cancer who are treated with adjuvant endocrine therapy (28-30). It has also been shown to predict benefit from adjuvant chemotherapy in patients with hormone receptor-positive breast cancer $(31,32)$.

Several studies have assessed the predictive value of RS for neoadjuvant/adjuvant endocrine treatment.

In NSABP B-14 trial, where adjuvant tamoxifen was tested in patients with node-negative hormone receptor-positive breast cancer, patients with low RS and intermediate RS showed significant benefit with tamoxifen while those with high RS did not (33). Patients with low RS showed 10-year distant recurrence-free survival (DRFS) of $85.9 \%$ with placebo and $93.1 \%$ with tamoxifen $(\mathrm{P}=0.039)$, those with intermediate $\mathrm{RS}$ showed that of $62.2 \%$ with placebo and $79.5 \%$ with tamoxifen $(\mathrm{P}=0.02)$ and those with high RS showed that of $68.7 \%$ with placebo and $70.3 \%$ with tamoxifen $(\mathrm{P}=0.82)$ (33). Although the result suggested the predictive value of RS for adjuvant tamoxifen benefit, the interaction between tamoxifen treatment and RS was marginal $(\mathrm{P}=0.06)$.

In ATAC trial, in which adjuvant anastrozole was compared with tamoxifen, the interaction between RS and treatment (anastrozole vs tamoxifen) was examined (28). No significant interaction was found regardless of nodal status, indicating that RS is not useful for selection of endocrine treatment, either aromatase inhibitor or tamoxifen (28).

In neoadjuvant settings, several studies have examined the predictive value of RS for clinical response (Table 1) (34-36). All the studies have shown that low RS is associated with better response rates while high RS is with worse response rates, indicating the usefulness of RS for prediction of response to neoadjuvant endocrine therapy. In addition, RS has been reported to be associated with breast conserving rate $(35,36)$. The combination of pre-treatment and post-treatment RSs has been reported to be able to predict early recurrence and late recurrence, separately, although a further validation is required (37). However, 20 $30 \%$ of the patients with high RS responded to neoadjuvant endocrine therapy while around $40 \%$ of the patients with low RS did not respond, suggesting that a better predictive tool is necessary to select neoadjuvant therapies in order to optimize treatment strategy.

\section{Breast cancer index (BCI)}

BCI was developed by utilizing two independently 
developed molecular assays including molecular grade index, which is a five-gene predictor of prognosis that recapitulates tumor grade and proliferation, and the two gene expression ratio, HOXB13/IL17BR (38-41). BCI has been shown to be a strong predictor for distant recurrence. In addition, BCI has been reported to be a potent predictor for late distant recurrence (42). Furthermore, in Trans-aTTom study, BCI [HOXB13/IL17BR ratio (H/I)] was shown to be associated with benefit from extended tamoxifen, namely 10 vs. 5 years of tamoxifen in patients with nodal involvement. Patients with $\mathrm{BCI}(\mathrm{H} / \mathrm{I})-\mathrm{High}$ derived a significant benefit from extended tamoxifen ( $\mathrm{HR}=0.35$; 95\% CI: $0.15-0.86 ; \mathrm{P}=0.0279$ ) while those with $\mathrm{BCI}(\mathrm{H} / \mathrm{I})$-Low showed no significant benefit ( $\mathrm{HR}=1.07 ; 95 \% \mathrm{CI}: 0.69-1.65 ; \mathrm{P}=0.77)$, indicating the clinical usefulness of BCI for application of extended use of endocrine therapy (43). Further validation studies are warranted.

\section{Conclusions}

A large number of studies have tried to identify biomarkers for endocrine therapy using samples from clinical trials or archives to optimize neoadjuvant/adjuvant endocrine therapies. Currently a limited number of markers are used as reliable marker in clinics. It is clear that further research is necessary to avoid unnecessary treatment and to optimize therapeutic agent and treatment duration. It is important to be aware that endocrine therapy affects not only cancer cells but also stromal cells and, thus, understanding stromal reaction including immune cells may help further development of biomarkers (44-47). In order to optimize neoadjuvant/adjuvant endocrine therapies, further research with multi-angle vision to understand biological meanings of endocrine response and resistance is required.

\section{Acknowledgments}

Funding: None.

\section{Footnote}

Provenance and Peer Review: This article was commissioned by the Guest Editors (Yutaka Yamamoto and Takayuki Ueno) for the series "Neoadjuvant/Adjuvant Treatment for Early Breast Cancer" published in Chinese Clinical Oncology. The article was sent for external peer review organized by the Guest Editors and the editorial office.

Conflicts of Interest: The author has completed the ICMJE uniform disclosure form (available at http://dx.doi. org/10.21037/cco-20-165). The series "Neoadjuvant/ Adjuvant Treatment for Early Breast Cancer" was commissioned by the editorial office without any funding or sponsorship. TU served as the unpaid Guest Editor of the series. TU reports personal fees from Chugai Pharmaceutical Co., Ltd., grants and personal fees from Eisai Co., Ltd., personal fees from AstraZeneca, personal fees from Taiho, personal fees from Novartis Pharma K.K., during the conduct of the study.

Ethical Statement: The author is accountable for all aspects of the work in ensuring that questions related to the accuracy or integrity of any part of the work are appropriately investigated and resolved.

Open Access Statement: This is an Open Access article distributed in accordance with the Creative Commons Attribution-NonCommercial-NoDerivs 4.0 International License (CC BY-NC-ND 4.0), which permits the noncommercial replication and distribution of the article with the strict proviso that no changes or edits are made and the original work is properly cited (including links to both the formal publication through the relevant DOI and the license). See: https://creativecommons.org/licenses/by-nc-nd/4.0/.

\section{References}

1. Rastelli F, Crispino S. Factors predictive of response to hormone therapy in breast cancer. Tumori 2008;94:370-83.

2. Davies C, Godwin J, Gray R, et al. Relevance of breast cancer hormone receptors and other factors to the efficacy of adjuvant tamoxifen: patient-level meta-analysis of randomised trials. Lancet 2011;378:771-84.

3. Kim C, Tang G, Pogue-Geile KL, et al. Estrogen receptor (ESR1) mRNA expression and benefit from tamoxifen in the treatment and prevention of estrogen receptor-positive breast cancer. J Clin Oncol 2011;29:4160-7.

4. Dowsett M, Allred C, Knox J, et al. Relationship between quantitative estrogen and progesterone receptor expression and human epidermal growth factor receptor 2 (HER-2) status with recurrence in the Arimidex, Tamoxifen, Alone or in Combination trial. J Clin Oncol 2008;26:1059-65.

5. Viale G, Regan MM, Maiorano E, et al. Prognostic and predictive value of centrally reviewed expression of estrogen and progesterone receptors in a randomized trial comparing letrozole and tamoxifen adjuvant therapy for postmenopausal early breast cancer: BIG 1-98. J Clin 
Oncol 2007;25:3846-52.

6. Bartlett JM, Brookes CL, Robson T, et al. Estrogen receptor and progesterone receptor as predictive biomarkers of response to endocrine therapy: a prospectively powered pathology study in the Tamoxifen and Exemestane Adjuvant Multinational trial. J Clin Oncol 2011;29:1531-8.

7. Ellis MJ, Ma C. Letrozole in the neoadjuvant setting: the P024 trial. Breast Cancer Res Treat 2007;105 Suppl 1:33-43.

8. Ueno T, Saji S, Masuda N, et al. Impact of clinical response to neoadjuvant endocrine therapy on patient outcomes: a follow-up study of JFMC34-0601 multicentre prospective neoadjuvant endocrine trial. ESMO Open 2018;3:e000314.

9. EBCTCG. Aromatase inhibitors versus tamoxifen in early breast cancer: patient-level meta-analysis of the randomised trials. Lancet 2015;386:1341-52.

10. Regan MM, Pagani O, Francis PA, et al. Predictive value and clinical utility of centrally assessed ER, PgR, and Ki67 to select adjuvant endocrine therapy for premenopausal women with hormone receptor-positive, HER2-negative early breast cancer: TEXT and SOFT trials. Breast Cancer Res Treat 2015;154:275-86.

11. Ellis MJ, Tao Y, Luo J, et al. Outcome prediction for estrogen receptor-positive breast cancer based on postneoadjuvant endocrine therapy tumor characteristics. J Natl Cancer Inst 2008;100:1380-8.

12. Ellis MJ, Suman VJ, Hoog J, et al. Ki67 Proliferation Index as a Tool for Chemotherapy Decisions During and After Neoadjuvant Aromatase Inhibitor Treatment of Breast Cancer: Results From the American College of Surgeons Oncology Group Z1031 Trial (Alliance). J Clin Oncol 2017;35:1061-9.

13. Goncalves R, DeSchryver K, Ma C, et al. Development of a Ki-67-based clinical trial assay for neoadjuvant endocrine therapy response monitoring in breast cancer. Breast Cancer Res Treat 2017;165:355-64.

14. Reinert T, Gonçalves R, Ellis MJ. Current Status of Neoadjuvant Endocrine Therapy in Early Stage Breast Cancer. Curr Treat Options Oncol 2018;19:23.

15. Toy W, Shen Y, Won H, et al. ESR1 ligand-binding domain mutations in hormone-resistant breast cancer. Nat Genet 2013;45:1439-45.

16. Robinson DR, Wu YM, Vats P, et al. Activating ESR1 mutations in hormone-resistant metastatic breast cancer. Nat Genet 2013;45:1446-51.

17. Zhang QX, Borg A, Wolf DM, et al. An estrogen receptor mutant with strong hormone-independent activity from a metastatic breast cancer. Cancer Res 1997;57:1244-9.

18. Liang X, Briaux A, Becette V, et al. Molecular profiling of hormone receptor-positive, HER2-negative breast cancers from patients treated with neoadjuvant endocrine therapy in the CARMINA 02 trial (UCBG-0609). J Hematol Oncol 2018;11:124.

19. Luen SJ, Asher R, Lee CK, et al. Association of Somatic Driver Alterations With Prognosis in Postmenopausal, Hormone Receptor-Positive, HER2-Negative Early Breast Cancer: A Secondary Analysis of the BIG 1-98 Randomized Clinical Trial. JAMA Oncol 2018;4:1335-43.

20. Razavi P, Chang MT, Xu G, et al. The Genomic Landscape of Endocrine-Resistant Advanced Breast Cancers. Cancer Cell 2018;34:427-38.e6.

21. Takeshita T, Yamamoto Y, Yamamoto-Ibusuki M, et al. Droplet digital polymerase chain reaction assay for screening of ESR1 mutations in 325 breast cancer specimens. Transl Res 2015;166:540-553.e2.

22. Reinert T, Ramalho S, de Vasconcelos VCA, et al. ESR1 Mutations Are Not a Common Mechanism of Endocrine Resistance in Patients With Estrogen Receptor-Positive Breast Cancer Treated With Neoadjuvant Aromatase Inhibitor Therapy. Front Oncol 2020;10:342.

23. Gelsomino L, Gu G, Rechoum Y, et al. ESR1 mutations affect anti-proliferative responses to tamoxifen through enhanced cross-talk with IGF signaling. Breast Cancer Res Treat 2016;157:253-65.

24. Beelen K, Opdam M, Severson TM, et al. PIK3CA mutations, phosphatase and tensin homolog, human epidermal growth factor receptor 2 , and insulin-like growth factor 1 receptor and adjuvant tamoxifen resistance in postmenopausal breast cancer patients. Breast Cancer Res 2014;16:R13.

25. Sabine VS, Crozier C, Brookes CL, et al. Mutational analysis of PI3K/AKT signaling pathway in tamoxifen exemestane adjuvant multinational pathology study. J Clin Oncol 2014;32:2951-8.

26. Ellis MJ, Ding L, Shen D, et al. Whole-genome analysis informs breast cancer response to aromatase inhibition. Nature 2012;486:353-60.

27. López-Knowles E, Segal CV, Gao Q, et al. Relationship of PIK3CA mutation and pathway activity with antiproliferative response to aromatase inhibition. Breast Cancer Res 2014;16:R68.

28. Dowsett M, Cuzick J, Wale C, et al. Prediction of risk of distant recurrence using the 21-gene recurrence score in node-negative and node-positive postmenopausal patients 
with breast cancer treated with anastrozole or tamoxifen: a TransATAC study. J Clin Oncol 2010;28:1829-34.

29. Paik S, Shak S, Tang G, et al. A multigene assay to predict recurrence of tamoxifen-treated, node-negative breast cancer. N Engl J Med 2004;351:2817-26.

30. Toi M, Iwata H, Yamanaka T, et al. Clinical significance of the 21-gene signature (Oncotype DX) in hormone receptor-positive early stage primary breast cancer in the Japanese population. Cancer 2010;116:3112-8.

31. Albain KS, Barlow WE, Shak S, et al. Prognostic and predictive value of the 21-gene recurrence score assay in postmenopausal women with node-positive, oestrogenreceptor-positive breast cancer on chemotherapy: a retrospective analysis of a randomised trial. Lancet Oncol 2010;11:55-65.

32. Paik S, Tang G, Shak S, et al. Gene expression and benefit of chemotherapy in women with node-negative, estrogen receptor-positive breast cancer. J Clin Oncol 2006;24:3726-34.

33. Paik S SS, Tang G et al., editor. Expression of the 21 genes in the Recurrence Score assay and tamoxifen clinical benefit in the NSABP study B-14 of node negative, estrogen receptor positive breast cancer. ASCO Annual Meeting; 2005.

34. Akashi-Tanaka S, Shimizu C, Ando M, et al. 21-Gene expression profile assay on core needle biopsies predicts responses to neoadjuvant endocrine therapy in breast cancer patients. Breast 2009;18:171-4.

35. Ueno T, Masuda N, Yamanaka T, et al. Evaluating the 21gene assay Recurrence $\operatorname{Score}(\mathrm{R})$ as a predictor of clinical response to 24 weeks of neoadjuvant exemestane in estrogen receptor-positive breast cancer. Int J Clin Oncol 2014;19:607-13.

36. Iwata H, Masuda N, Yamamoto Y, et al. Validation of the 21-gene test as a predictor of clinical response to neoadjuvant hormonal therapy for ER+, HER2-negative breast cancer: the TransNEOS study. Breast Cancer Res Treat 2019;173:123-33.

37. Ueno T, Saji S, Masuda N, et al. Changes in Recurrence Score by neoadjuvant endocrine therapy of breast cancer and their prognostic implication. ESMO Open 2019;4:e000476.

Cite this article as: Ueno T. Biomarkers of neoadjuvant/ adjuvant endocrine therapy for ER-positive/HER2-negative breast cancer. Chin Clin Oncol 2020;9(3):35. doi: 10.21037/ cco-20-165
38. Jerevall PL, Ma XJ, Li H, et al. Prognostic utility of HOXB13:IL17BR and molecular grade index in earlystage breast cancer patients from the Stockholm trial. Br J Cancer 2011;104:1762-9.

39. Ma XJ, Hilsenbeck SG, Wang W, et al. The HOXB13:IL17BR expression index is a prognostic factor in early-stage breast cancer. J Clin Oncol 2006;24:4611-9.

40. Ma XJ, Wang Z, Ryan PD, et al. A two-gene expression ratio predicts clinical outcome in breast cancer patients treated with tamoxifen. Cancer Cell 2004;5:607-16.

41. Zhang Y, Schnabel CA, Schroeder BE, et al. Breast Cancer Index Identifies Early Stage ER+ Breast Cancer Patients at Risk for Early and Late Distant Recurrence. Clin Cancer Res 2013;19:4196-205.

42. Sgroi DC, Sestak I, Cuzick J, et al. Prediction of late distant recurrence in patients with oestrogen-receptorpositive breast cancer: a prospective comparison of the breast-cancer index (BCI) assay, 21-gene recurrence score, and IHC4 in the TransATAC study population. Lancet Oncol 2013;14:1067-76.

43. Bartlett JMS, Sgroi DC, Treuner K, et al. Breast Cancer Index and prediction of benefit from extended endocrine therapy in breast cancer patients treated in the Adjuvant Tamoxifen-To Offer More? (aTTom) trial. Ann Oncol 2019;30:1776-83.

44. Generali D, Bates G, Berruti A, et al. Immunomodulation of FOXP3 + regulatory $\mathrm{T}$ cells by the aromatase inhibitor letrozole in breast cancer patients. Clin Cancer Res 2009; $15: 1046-51$.

45. Ueno T, Masuda N, Kamigaki S, et al. Differential Involvement of Autophagy and Apoptosis in Response to Chemoendocrine and Endocrine Therapy in Breast Cancer: JBCRG-07TR. Int J Mol Sci 2019;20:984.

46. Ueno T, Saji S, Sugimoto M, et al. Clinical significance of the expression of autophagy-associated marker, beclin 1 , in breast cancer patients who received neoadjuvant endocrine therapy. BMC Cancer 2016;16:230.

47. Ueno T, Utsumi J, Toi M, et al. Characteristic Gene Expression Profiles of Human Fibroblasts and Breast Cancer Cells in a Newly Developed Bilateral Coculture System. Biomed Res Int 2015;2015:960840. 